This is a preprint of the following accepted manuscript, the published article may differ from the preprint.

Zhao, W. and Kapania, R. K., "Skin-Stringer Assembly using Radial Basis Functions for Curvilinearly Stiffened Panels," AIAA Journal, 2020, DOI: 10.2514/1. J059364

\title{
Skin-Stringer Assembly using Radial Basis Functions for Curvilinearly Stiffened Panels
}

\author{
Wei Zhao * and Rakesh K. Kapania ${ }^{\dagger}$ \\ Virginia Polytechnic Institute and State University, Blacksburg, VA, 24061
}

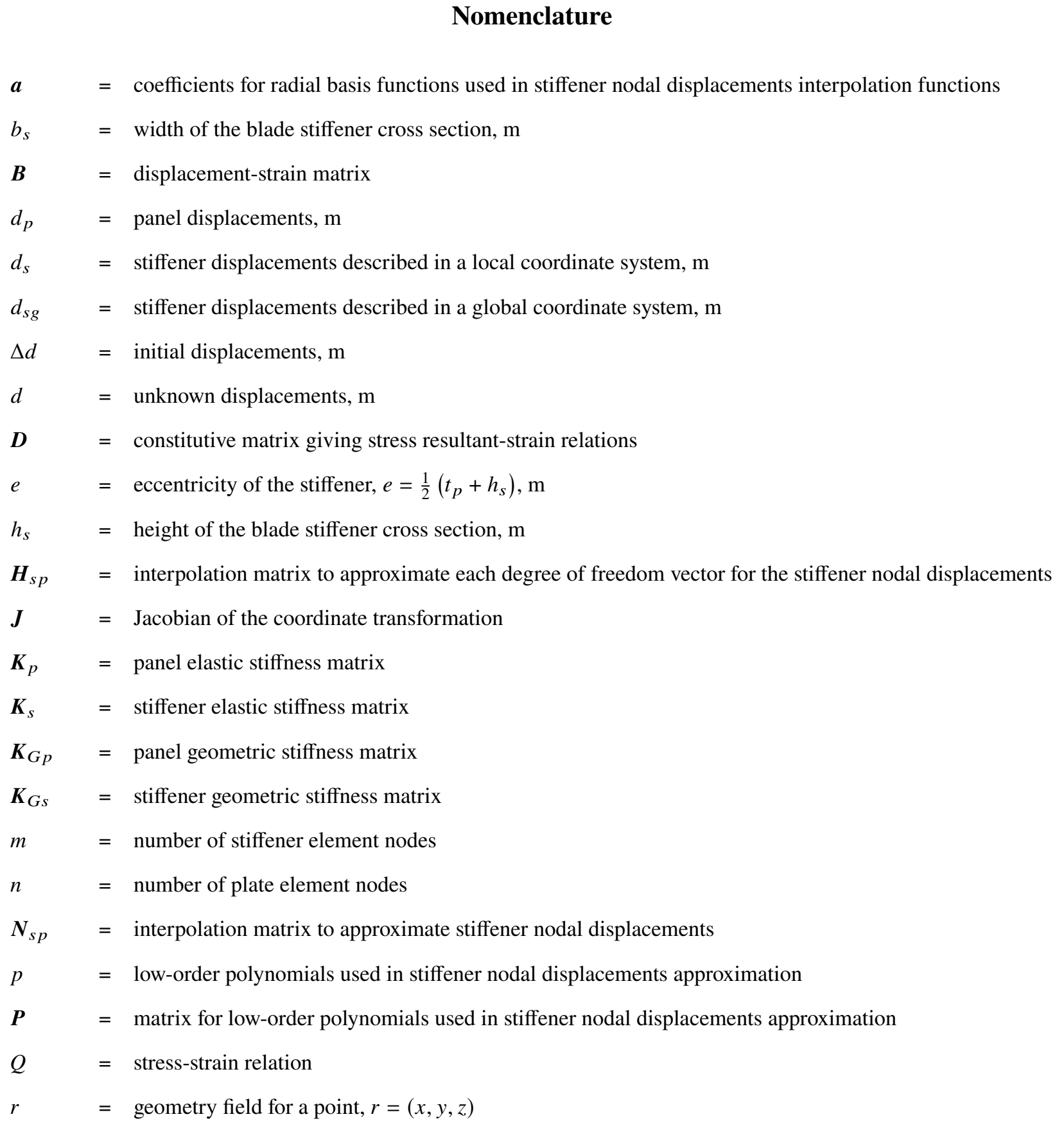

*Research Scientist, Kevin T. Crofton Department of Aerospace and Ocean Engineering, weizhao@ vt.edu, AIAA Member

${ }^{\dagger}$ Mitchell Professor, Kevin T. Crofton Department of Aerospace and Ocean Engineering, AIAA Fellow 


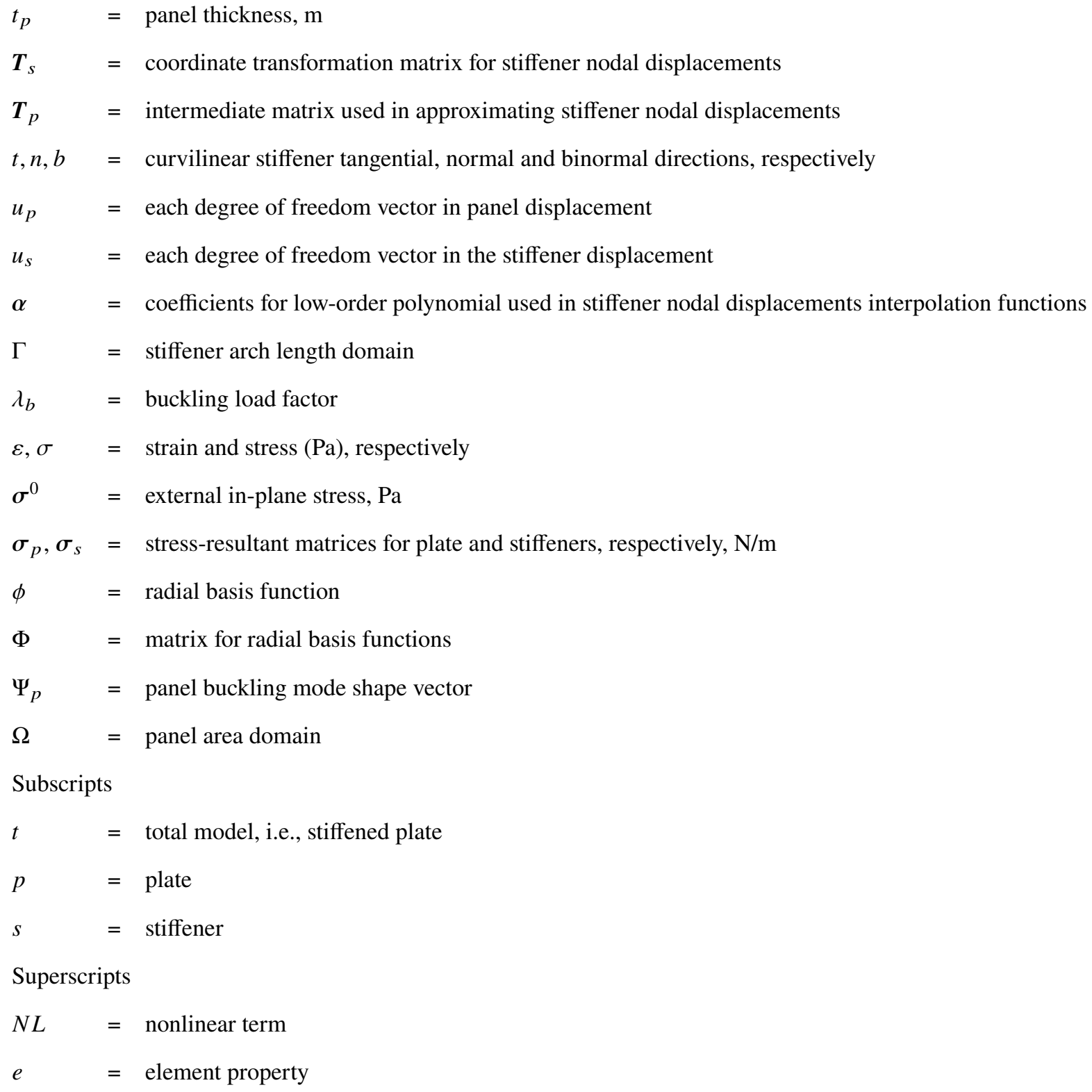

\section{Introduction}

Recent studies on design optimization of aircraft panels using curvilinear stiffeners reveal that it is possible to improve both the buckling and vibration responses of stiffened structures by varying the stiffener's shape, which change the stiffness as well as the buckling and vibration mode shapes [1-3]. Due to the enhanced tailorability of stiffness distribution of stiffened panels, provided by curvilinear stiffeners and curvilinear fiber paths for a plate; Stanford and Jutte [4] studied the aircraft wing box design simultaneously using both curvilinear stiffeners and tow-steered composites in a wing panel design. The optimization results showed that this simultaneous use of curvilinear fibers and curvilinear stiffeners leads to a larger weight reduction as compared to the design that uses such stiffening individually. Singh 
and Kapania [5] studied the buckling maximization for a composite panel with both curvilinear fibers and curvilinear stiffeners. Their study too showed a substantial benefit of using the two technologies simultaneously for maximizing the panel buckling loads. Zhao and Kapania [6] found that the buckling temperature increases when using curvilinear stiffeners to tailor the buckling mode shape.

Many efforts have been made towards performing structural analysis and design optimization of curvilinearly stiffened plates. Kapania et al. [7] developed an optimization framework by using commercial software, MSC PATRAN and MSC NASTRAN, for analysis and optimization of curvilinearly stiffened plates. An improved optimization framework, named EBF3PanelOpt, was later developed by Mulani et al. [8] for studying both curvilinearly stiffened flat and curved panels. A challenging task in this optimization framework was to mesh curvilinearly stiffened structure when there are multiple stiffeners. For finite element assemblage of stiffeners and plate elements, the nodes for stiffeners and plate should coincide at plate-stiffener and stiffener-stiffener interfaces. However, the meshing often failed in satisfying such element nodal coincidences when there are multiple stiffeners or the stiffeners have complex geometries. A fine mesh was often used to avoid meshing failure. It was suggested that one uses at the most six stiffeners for optimizing the curvilinear stiffeners when using EBF3PanelOpt so as to avoid excessive mesh failure during optimization, which may result in a reduced design space during the stiffener's shape optimizing.

To overcome the meshing failure in the EBF3PanelOpt, a new technology, named glue contact [9], available in MSC PATRAN/NASTRAN was next employed. Glue contact uses multipoint constraints to connect two surfaces together without the need of satisfying the nodal overlaps at the interface between the two structures. Singh et al. [3] employed this technology in studying the buckling load maximization of curvilinearly stiffened shells. In their studies, the common nodes at the plate-stiffeners interfaces during meshing are not considered but they are still considered at the stiffener-stiffener interfaces. The mesh generation for curvilinearly stiffened shells becomes very complex when there are multiple stiffeners or the stiffener's shape becomes complex during optimization. When modeling the stiffeners as beam elements, the glue contact may influence the accuracy of the analysis results because it is very sensitive to the offset between the stiffener's centroid and the plate's median plane [10]. Additionally, both semi-analytical and mesh-less methods were developed for buckling and vibration analysis of curvilinearly stiffened plates by Tamijani and Kapania [11-13], Vescovini et al. [14, 15] and Alanbay et al. [16]. For practical aircraft panels, which have both complex geometry and complex boundary conditions, it is not efficient to use these semi-analytical methods as one needs to find admissible trial functions to satisfy those complex essential boundary conditions.

An innovative finite element method was later developed for studying the buckling and vibration of curvilinearly stiffened plates by Shi and Kapania [17, 18] and Zhao and Kapania [1, 2, 6]. These works used separate modeling in developing finite element models for the plate and the stiffeners. The plate and the stiffeners are modeled by using Mindlin plate and Timoshenko beam theories, respectively. To assemble the stiffeners' elastic stiffness, mass and geometric stiffness matrices to those for the plate, the element nodal displacements for the stiffeners are approximated 
by those for the plate. This is accomplished through the displacement compatibility conditions at the stiffener/plate interfaces by employing isoparametric plate element shape functions. The displacement compatibility condition at the stiffener-stiffener interfaces is satisfied automatically as they are approximated using the same plate element nodal displacements. Since only the converged mesh for the plate is used, no meshing failures were observed when using this method during the stiffener's shape optimization. This approach obviates the need for a repeated meshing of the curvilinearly stiffened plate when there is any change in the stiffener's placement or the stiffener's shape. Also, this approach works for any shape and any number of stiffeners for stiffened plates during optimization. Additionally, the alternative modeling approach shows its benefits in modeling the plate with tow-steered laminates. Traditional finite element method needs to re-compute the fiber path orientation for each layer of each plate element due to a remeshing when there is any change in the stiffener's shape. The aforementioned approach does not affect the plate mesh and hence there is no need to re-evaluate the element fiber path orientations when any change in the stiffener's shape is made.

However, to use this method, one needs to know the plate element IDs for all stiffener element nodes a priori. Armed with that knowledge, one can compute the plate natural coordinates, used in describing the plate element shape function, corresponding to the stiffener element nodes at the plate elements. These natural coordinates are obtained from the high-order polynomials that are used to approximate the stiffener node coordinates in terms of those known plate element nodal coordinates. The natural coordinates are then used to determine the stiffener element nodal displacements in terms of the plate element nodal displacements by using the isoparametric plate shape functions. Furthermore, this processing may also take significant computational time depending on the number of both the stiffeners and the stiffener element nodes.

Instead of being approximated from using the isoparametric plate element shape functions, the stiffener nodal displacement are being approximated in this paper from the global plate displacement that are represented using radial basis functions (RBFs). This representation is inspired by ours, and others, experience with aero/structure coupling for approximating the aerodynamic model displacement from the structural motion [19, 20] and the work by Wei et al. [21] on truss structure optimization. Wei et al [21] used the $C^{2}$ Wendland functions based radial basis functions to approximate the truss bar nodal displacement in terms of the background mesh node displacements. The background rectangular mesh assumes a weak, ghost material. The truss structure optimization was conducted to determine the optimal truss members layout subjected to a tip load, a different application from the present study. Wei et al's method does not require the bar elements in a truss structure to be connected during the structural layout optimization, which allows to optimize the size, shape and topology of the truss members cocurrently for an efficient structural design.

In the present study, the RBFs based stiffener displacement approximation avoids searching the plate element IDs for each stiffener element node and obviates the need to compute the natural coordinates for all stiffener nodes as used in the isoparametric shape functions based approximations. The continuously globally-supported RBFs enables all stiffener element nodal displacements to be approximated by using all the plate element nodal displacements, which improves 
the structural analysis efficiency of stiffened plates with complex shape stringers for use in practical applications.

There are some other polynomials that have been used to represent the global plate displacement. For example, Byun and Kapania [22] used both Chebyshev polynomials and a class of globally orthogonal polynomials to approximate the global structural displacements for composite plates from the finite element data. The coefficients of these polynomials were obtained using the method of least squares by minimizing the square of difference between the finite element values and the global approximations. The approximated global displacements are used for interlaminar stress prediction through the plate thickness that required higher-order derivatives, which are not available from the low-order finite element shape functions. Although these polynomials demonstrate their advantages in displacement approximations for the subsequent stress recovery, they require a time-consuming additional, intermediate step to determine the coefficients for the polynomials. The requisite optimization for determining these coefficients may take significant computational time when applying these polynomials for approximating the displacements for multiple stiffeners during the optimization.

The technical note is organized as follows: Section $\Pi$ presents expressions for the strain energy and the potential of the in-plane loads, respectively, for the plate and the stiffeners for the curvilinearly stiffened composite plate. Section III presents the stiffener displacement approximation in terms of the plate displacements by using radial basis functions. The present approach is verified by employing it on a previously studied problem of buckling of a stiffened, variable angle tow (VAT) laminated panel with four curvilinear stiffeners, and subjected to an end-shortening. The details of the example and the results are presented in Section $\mathrm{IV}$ The last section, Section $\mathrm{V}$, concludes the present work.

\section{Formulations}

\section{A. Motion of Stiffened VAT Laminated Plate}

The relevant formulations for studying buckling of stiffened composite plates with curvilinear stiffeners (see Fig. 1) have been presented in our previous work [6, 23]. For brevity, those formulations will not be shown here. The required energy expressions are presented here to show the derivation of the governing equation for buckling analysis. The plate is modeled as plate elements and the stiffener is modeled as Timoshenko beam when using the finite element method.

The formulations used in the buckling analysis involve pre-buckling and buckling analyses. The pre-buckling analysis is to determine the stress-resultant distribution for the stiffened plates subject to external loads. The stress-resultants are used to compute the geometric stiffness matrices for both the plate and the stiffeners in the subsequent buckling analysis. The strain energy, $U_{t}$, for the curvilinearly stiffened, VAT composite panel is same in pre-buckling and buckling analysis, 


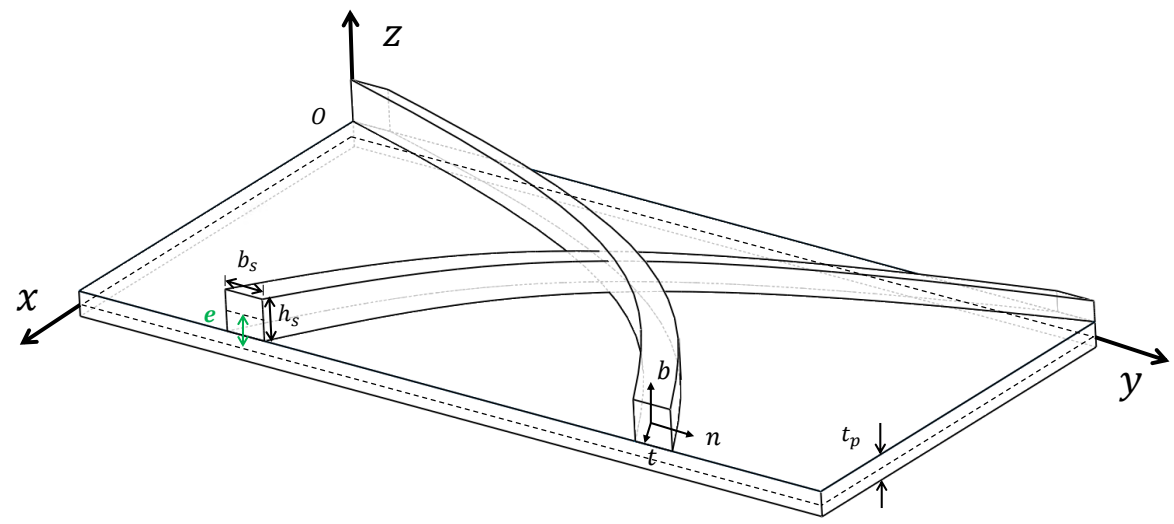

Fig. 1 Schematic of curvilinearly stiffened plate

which is obtained by summing the strain energy for the plate, $U_{p}$, and the strain energy for the stiffeners, $U_{s}$ [1]:

$$
\begin{aligned}
U_{t} & =U_{p}+U_{s} \\
U_{p} & =\frac{1}{2} \iint_{\Omega} \boldsymbol{d}_{p}^{T} \boldsymbol{B}_{p}^{T} \boldsymbol{D}_{p}^{T} \boldsymbol{B}_{p} \boldsymbol{d}_{p} \mathrm{~d} \Omega \\
U_{s} & =\frac{1}{2} \int_{\Gamma} \boldsymbol{\varepsilon}_{s}^{T} \boldsymbol{D}_{s}^{T} \boldsymbol{\varepsilon}_{s} \mathrm{~d} \Gamma=\frac{1}{2} \int_{\Gamma} \boldsymbol{d}_{p}^{T} \boldsymbol{N}_{s p}^{T} \boldsymbol{T}_{s}^{T} \boldsymbol{B}_{s}^{T} \boldsymbol{D}_{s} \boldsymbol{B}_{s} \boldsymbol{T}_{s} \boldsymbol{N}_{s p} \boldsymbol{d}_{p} \mathrm{~d} \Gamma
\end{aligned}
$$

where $\boldsymbol{d}_{p}$ is the generalized displacement field for the point at the middle plane of the composite panel; $\boldsymbol{B}_{p}$ is the plate's strain-displacement relation matrix; $\boldsymbol{D}_{p}$ is the constitutie matrix for the plate stress resultant-strain relation. The matrix $\boldsymbol{T}_{s}$ is a transformation matrix which relates the stiffener displacement field, $\boldsymbol{d}_{s}$, described in the local coordinate system tnb and the stiffener displacement field, $\boldsymbol{d}_{s g}$, described in the global coordinate system $x y z, \boldsymbol{d}_{s}=\boldsymbol{T}_{s} \boldsymbol{d}_{s g}$. Matrix $\boldsymbol{B}_{s}$ is the displacement-strain matrix for the stiffener. The expressions for these matrices can be found in Ref. [1]. The stress-resultant and strain constitute matrix for the composite stiffeners, $D_{s}$, is given in Appendix A. The approximation matrix $\boldsymbol{N}_{s p}$ is used to approximate the stiffener displacements in terms of the plate displacements by using radial basis functions, which is shown in Section III

The potential of external load used in static analysis (pre-buckling analysis) is not shown here for brevity. In buckling analysis, the potential of external in-plane loads, $W_{t}$, for the stiffened plate due to the geometric nonlinearity can be obtained by summing the potential, $W_{p}$, of the panel due to an in-plane stress, $\sigma^{0}$, and the potential for the stiffener, $W_{s}$, subjected to an axial stress, $\sigma_{t}$, is

$$
\begin{aligned}
& W_{t}=W_{p}+W_{s} \\
& W_{p}=-\iiint_{V}\left(\sigma^{0}\right)^{T} \varepsilon_{p}^{N L} \mathrm{~d} V=-\frac{1}{2} \iint_{\Omega} \boldsymbol{d}_{p}{ }^{T}\left(\boldsymbol{B}_{p}^{N L}\right)^{T} \boldsymbol{\sigma}_{p} \boldsymbol{B}_{p}^{N L} \boldsymbol{d}_{\boldsymbol{p}} \mathrm{d} \Omega \\
& W_{s}=-\frac{1}{2} \int_{\Gamma} \boldsymbol{d}_{p}^{T} \boldsymbol{N}_{s p}^{T} \boldsymbol{T}_{s}^{T}\left(\boldsymbol{B}_{s}^{N L}\right)^{T} \boldsymbol{\sigma}_{s} \boldsymbol{B}_{s}^{N L} \boldsymbol{T}_{s} \boldsymbol{N}_{s p} \boldsymbol{d}_{p} \mathrm{~d} \Gamma
\end{aligned}
$$


where $\varepsilon_{p}^{N L}$ is a vector for the nonlinear terms of the in-plane strains. The in-plane stress vector, $\sigma^{0}$, for the panel is obtained by solving a static analysis of the plate subjected to in-plane end-shortening in the present study; $\sigma_{p}$ and $\sigma_{s}$ are stress-resultants matrices for the plate and the stiffeners, respectively. The matrices $\boldsymbol{B}_{p}^{N L}$ and $\boldsymbol{B}_{s}^{N L}$ are nonlinear in-plane strain relation matrices for the plate and the stiffeners, respectively. For brevity, the expressions for these matrices are not shown, which can be found in previous work [1].

\section{B. Finite Element Buckling Analysis}

For a linear buckling analysis, a pre-buckling analysis is conducted to obtain the in-plane stress-resultants in both the panel and the stiffeners. The geometric stiffnesses for both the plate and the stiffeners are computed in terms of these in-plane stress-resultants. For a stiffened panel subjected to an in-plane end-shortening, $\Delta \boldsymbol{d}$, a static analysis is conducted as:

$$
[\boldsymbol{K}]\left\{\boldsymbol{d}_{p}\right\}=\left[\begin{array}{cc}
\left(\boldsymbol{K}_{11}\right)_{k \times k} & \left(\boldsymbol{K}_{12}\right)_{k \times l} \\
\left(\boldsymbol{K}_{21}\right)_{l \times k} & \left(\boldsymbol{K}_{22}\right)_{l \times l}
\end{array}\right]\left\{\begin{array}{c}
\Delta \boldsymbol{d} \\
\boldsymbol{d}
\end{array}\right\}=\left\{\begin{array}{c}
\boldsymbol{f} \\
\mathbf{0}
\end{array}\right\}
$$

where $k$ is the number of degrees of freedom with known displacements and $l$ is the number of unknown displacements.

The displacement field for the plate is summarized as:

$$
\left\{d_{p}\right\}=\left\{\begin{array}{c}
\Delta d \\
d
\end{array}\right\}
$$

where the expression for the unknown displacement, $\boldsymbol{d}$ can be obtained based on Eq. (3)

$$
\boldsymbol{d}=-\boldsymbol{K}_{22}^{-1} \boldsymbol{K}_{21} \Delta \boldsymbol{d}
$$

The in-plane stresses for both the plate and the stiffeners are solved by using following equations:

$$
\boldsymbol{\sigma}_{p}=\boldsymbol{Q}_{p} \boldsymbol{\varepsilon}_{p}=\boldsymbol{Q}_{p} \boldsymbol{B}_{p} \boldsymbol{d}_{p} \quad \text { and } \quad \boldsymbol{\sigma}_{t}=\boldsymbol{Q}_{s} \boldsymbol{\varepsilon}_{t}=\boldsymbol{Q}_{s} \boldsymbol{B}_{s} \boldsymbol{T}_{s} \boldsymbol{N}_{s p} \boldsymbol{d}_{p}
$$

where $\boldsymbol{Q}_{p}$ and $\boldsymbol{\varepsilon}_{p}$ have different values when they are computed at different Gaussian points for each element in the VAT laminates. In this work, the averaged stress for each layer of the laminates are used to compute the geometric stiffness matrices for the plate and the stiffeners. The buckling load factor, $\lambda_{b}$, can be obtained through an eigenvalue analysis:

$$
\left[\left(\boldsymbol{K}_{p}+\boldsymbol{K}_{s}\right)+\lambda_{b}\left(\boldsymbol{K}_{G p}+\boldsymbol{K}_{G s}\right)\right]\left\{\boldsymbol{\Psi}_{p}\right\}=\mathbf{0}
$$


where $\boldsymbol{\Psi}_{p}$ is the plate's buckling mode shape; $\boldsymbol{K}_{p}$ and $\boldsymbol{K}_{s}$ are the elastic stiffness matrices for the panel and the stiffeners, respectively; $\boldsymbol{K}_{G p}$ and $\boldsymbol{K}_{G s}$ are the geometric stiffness matrices for the panel and the stiffeners, respectively. The element stiffness matrices, $\boldsymbol{K}_{p}^{e}$ and $\boldsymbol{K}_{s}^{e}$, and the element geometric stiffness matrices, $\boldsymbol{K}_{G p}^{e}$ and $\boldsymbol{K}_{G s}^{e}$, respectively, are

$$
\begin{aligned}
& \boldsymbol{K}_{p}^{e}=\int_{-1}^{+1} \int_{-1}^{+1} \boldsymbol{B}_{p}^{T} \boldsymbol{D}_{p}^{T} \boldsymbol{B}_{p} \operatorname{det} \boldsymbol{J}_{p} \mathrm{~d} \xi \mathrm{d} \eta \\
& \boldsymbol{K}_{s}^{e}=\left(\boldsymbol{N}_{s p}^{e}\right)^{T}\left(\int_{-1}^{+1} \boldsymbol{T}_{s}^{T} \boldsymbol{B}_{s}^{T} \boldsymbol{D}_{s}^{T} \boldsymbol{B}_{s} \boldsymbol{T}_{s} \operatorname{det} \boldsymbol{J}_{s} \mathrm{~d} \xi\right) \boldsymbol{N}_{s p}^{e} \\
& \boldsymbol{K}_{G p}^{e}=\int_{-1}^{+1} \int_{-1}^{+1}\left(\boldsymbol{B}_{p}^{N L}\right)^{T} \boldsymbol{\sigma}_{p} \boldsymbol{B}_{p}^{N L} \operatorname{det} \boldsymbol{J}_{p} \mathrm{~d} \xi \mathrm{d} \eta \\
& \boldsymbol{K}_{G s}^{e}=\left(\boldsymbol{N}_{s p}^{e}\right)^{T}\left(\int_{-1}^{+1} \boldsymbol{T}_{s}^{T}\left(\boldsymbol{B}_{s}^{N L}\right)^{T} \boldsymbol{\sigma}_{s} \boldsymbol{B}_{s}^{N L} \boldsymbol{T}_{s} \operatorname{det} \boldsymbol{J}_{s} \mathrm{~d} \xi\right) \boldsymbol{N}_{s p}^{e}
\end{aligned}
$$

where $\boldsymbol{J}_{p}$ and $\boldsymbol{J}_{s}$ are Jacobians for the panel and the stiffener, respectively; $\boldsymbol{N}_{s p}^{e}$ is use to approximate nodal displacements for each stiffener beam element, which can be obtained from the global stiffener element nodal displacement approximation matrix, $\boldsymbol{N}_{s p}$.

\section{Stiffener Displacements Approximation}

Previous work [1, 17] used piece-wise functions to approximate the stiffener element nodal displacements in terms of the plate displacements. The piece-wise functions are based on the isoparametric shape functions for the plate element, which is defined in a single plate element. Figure 2 shows an example of the finite element model for a stiffened plate with two curved stiffeners. A representative plate element with stiffener beam element is chosen to briefly explain the previous work using isoparametric shell element shape functions to approximate stiffener nodal displacements.
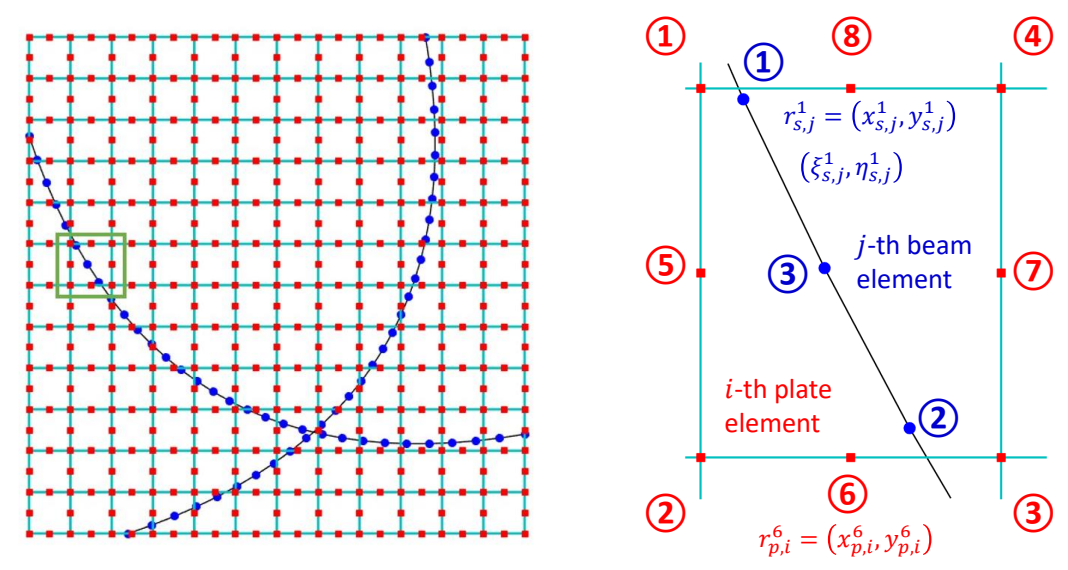

Fig. 2 Separate modeling for curvilinear stiffeners and plate

Considering that the $j$-th stiffener beam element passes through the $i$-th plate element as shown in Fig. 2 Both the displacement and geometry expressions for the three nodes of the $j$-th beam element can be approximated in terms of the displacement and geometry for the eight nodes of the $i$-th plate element when using the isoparametric 
shape functions in the finite element analysis. The displacement and the geometry coordinate for each element node ( $m=1,2,3)$ of the $j$-th beam element can be expressed as:

$$
\begin{aligned}
r_{s, j}^{m} & =\sum_{k=1}^{8} N_{p, i}^{k}\left(\xi_{s, j}^{m}, \eta_{s, j}^{m}\right) r_{p, i}^{k} \\
d_{s g, j}^{m} & =\sum_{k=1}^{8} N_{p, i}^{k}\left(\xi_{s, j}^{m}, \eta_{s, j}^{m}\right) d_{p, i}^{k}
\end{aligned}
$$

where $N_{p}$ is the shape function for an eight-noded plate element. The superscript $k$ for $N_{p}$ is the node ID for each plate element, and $i$ is used to label the plate element where the stiffener beam element node is located. The natural coordinates $\left(\xi_{s, j}^{m}, \eta_{s, j}^{m}\right)$ for the $m$-th node of the $j$-th beam element are shown in Fig. 2. Since the geometry fields for the plate $\left\{\boldsymbol{r}_{p}\right\}$ and the stiffeners $\left\{\boldsymbol{r}_{s}\right\}$ are known, the natural coordinates $\left(\xi_{s, j}^{m}, \eta_{s, j}^{m}\right)$ can be obtained through the geometric approximations for isoparametric elements by using Eq. 9a. The solved natural coordinates are then used to approximate the stiffener displacements in Eq. 9b). Repeat the process for all stiffener beam element nodes, all nodal displacements for the stiffeners can be obtained and written in a matrix form as:

$$
\left\{\boldsymbol{d}_{s g}\right\}=\left[\boldsymbol{N}_{s p}\right]\left\{\boldsymbol{d}_{p}\right\}
$$

Note that a curvilinear coordinate system is used to express the stiffener motion. The stiffener displacement field described in the global coordinate system, $\left\{\boldsymbol{d}_{s g}\right\}$, is approximated in terms of the plate displacements, which can be transformed to that described in the stiffener curvilinear coordinate system by using a transformation matrix, $\left[\boldsymbol{T}_{s}\right]$. To obtain the approximation matrix, $N_{s p}$, one needs to know all the plate element IDs a priori where the stiffener nodes are located (see Fig. 2). After that, one needs to solve Eq. 9a for natural coordinates: $\left(\xi_{s, j}^{m}, \eta_{s, j}^{m}\right)$ for each stiffener beam element node. When there are multiple stiffeners and many plate elements, the pre-processing required in determining the plate element IDs and computing the natural coordinates for all stiffener element nodes during the stiffener shape optimization is computationally inefficient. The approach presented here eliminates this pre-processing.

Based on the aero/structure coupling study [19, 24] and Wei et al's work [21], and noticing that the stiffeners are always located within the panel, a globally continuous and differentiable radial basis function (RBF) is used as interpolation function for approximating the stiffener nodal displacements in terms of the plate nodal displacements. The RBFs based approximation does not need us to find the plate element IDs and to compute the natural coordinates for given stiffener nodes. For each stiffener element node, the displacement field, using radial basis function, $\phi$, and a polynomial, $p$, can be expressed as a general form:

$$
u_{s g}\left(r_{s}\right)=\sum_{j=1}^{n} a_{j} \phi\left(\left\|r_{s}-r_{p, j}\right\|\right)+p\left(r_{s}\right)
$$


where $a_{j}$ is the coefficient for the $j$-th radial basis function, and $u_{s g}\left(r_{s}\right)$ is the stiffener nodal displacement described in the global coordinate system, representing the same degree of freedom; $r$ represents the geometry field for a point, $r=(x, y, z) ; r_{s}$ is stiffener geometry field for a point in $R^{3}$, representing here a stiffener element node coordinate; $n$ is the number of the plate element nodes; $r_{p, j}$ are the center points for the RBFs, representing the $j$-th node coordinate for the plate element in the present work; $\phi\left(\left\|r_{s}-r_{p, j}\right\|\right)$ is the basis function with respect to the Euclidean distance. There are many different types of radial basis functions as summarized by Buhmann [25] that one can employ. When the chosen basis function, $\phi$, is not positive definite, the lower-order polynomials are required [26]. The function $p(r)$ are monomial terms, which are lower-order polynomials as compared to the basis functions, and include the lower-order polynomials and a constant that are not present in the radial basis functions [24, 26- -28$]$.

The coefficients, $a_{j}$, and polynomial, $p(r)$, given in Eq. (11) are determined using:

$$
\begin{aligned}
& u_{s g}\left(r_{p, j}\right)=u_{p, j} \\
& \sum_{j=1}^{n} a_{j} q\left(r_{p, j}\right)=0
\end{aligned}
$$

Here, Eq. (12a) shows that the interpolated displacement obtained using the RBFs at the plate element nodes should be same as the panel element node displacement, $u_{s g}\left(r_{p, j}\right)=u_{p, j}$. Equation (12b) represents the condition of orthogonality that should be satisfied for any polynomial $q$ which has a degree less than or equal to that of polynomial $p$. Note that $\boldsymbol{u}_{p}$ and $\boldsymbol{u}_{s}$ represent a degree of freedom in the global displacement field for the plate, $\boldsymbol{d}_{p}$, and for the stiffeners, $\boldsymbol{d}_{s g}$, such as the transverse displacement.

In this study, a compactly supported radial basis function developed by Wendland [29], the one with $C^{2}$ continuity, because of its positive definite property and the sparsity of the resulting system of equations matrix, is used. Also, the $C^{2}$ Wendland basis function has high order polynomials that are equivalent to the 8-noded isoparametric shell element's shape functions used in this work. Additionally, the $C^{2}$ Wendland basis function chooses the neighbouring plate element nodes for approximating the stiffener nodal displacement by using a support radius, which is similar to the isoparametric shell element shape functions based displacement approximations but it behaves globally continuous. The expression of this RBF is shown in Eq. (13) and its details can be found in Ref. [29]. The support radius, $r_{\text {max }}$, used in $C^{2}$ Wendland RBF is 2.2 times the minimal element size of the plate as suggested by Wei et al. [21]. A converged mesh is used for the minimal element size of the plate in the present work. The determination of the support radius, $r_{\max }$, is only needed to study once when there is no change in the plate finite element model. The $C^{2}$ Wendland basis function is expressed as:

$$
\phi(\|\boldsymbol{x}\|)= \begin{cases}\left(1-\|\boldsymbol{x}\| / r_{\max }\right)_{+}^{4} \cdot\left(4\|\boldsymbol{x}\| / r_{\max }+1\right) & \text { if }\|\boldsymbol{x}\| \leq r_{\max } \\ 0 & \text { otherwise }\end{cases}
$$


The order of polynomial $p$ given in Eq. (11) depends on the selected basis function, $\phi$. A unique interpolation exists if the basis function is a conditionally positive definite function [24]. In this study, the chosen basis functions have polynomials order of $m \geq 2$. A linear polynomial, $p\left(r_{s}\right)$, is chosen as: $\beta_{0}+\beta_{1} x+\beta_{2} y+\beta_{3} z$. For a 2D structure problem, the linear polynomial could be $\beta_{0}+\beta_{1} x+\beta_{2} y$. For completeness, the following derivations consider to use 3D structures to explain the RBFs for stiffener displacement approximation. Based on the previous work using isoparametric shell element shape functions for stiffener displacement approximation, given in Eq. 99, the linear and constant terms were considered. Note that $C^{2}$ Wendland RBF has no linear terms. Therefore, for comparison, a lower-order polynomial, $p(r)$, is considered in the present RBF function. The determination of the coefficients for the RBFs is explained in the following. The following derivations considered the linear terms in the RBFs based displacement approximations. The results without considering linear terms are also studied in Section IV for comparison.

Equation (12b) leads to following equations to satisfy the condition of orthogonality for the lower-order polynomials:

$$
\begin{aligned}
& \sum_{j=1}^{n} a_{j} \beta_{0}=0 \\
& \sum_{j=1}^{n} a_{j} \beta_{1} x_{j}=0 \\
& \sum_{j=1}^{n} a_{j} \beta_{2} y_{j}=0 \\
& \sum_{j=1}^{n} a_{j} \beta_{3} z_{j}=0
\end{aligned}
$$

Equations (14) and (12a) can be combined in a matrix form:

$$
\left[\begin{array}{cc}
\mathbf{0} & \boldsymbol{P}_{p}^{T} \\
\boldsymbol{P}_{p} & \boldsymbol{\Phi}_{p p}
\end{array}\right]\left\{\begin{array}{c}
\boldsymbol{\beta} \\
\boldsymbol{a}
\end{array}\right\}=\left\{\begin{array}{c}
\mathbf{0} \\
\boldsymbol{u}_{p}
\end{array}\right\}
$$

where

$$
\boldsymbol{\Phi}_{p p}=\left[\begin{array}{cccc}
\phi\left(\left\|r_{p, 1}-r_{p, 1}\right\|\right) & \phi\left(\left\|r_{p, 1}-r_{p, 2}\right\|\right) & \ldots & \phi\left(\left\|r_{p, 1}-r_{p, n}\right\|\right) \\
\phi\left(\left\|r_{p, 2}-r_{p, 1}\right\|\right) & \phi\left(\left\|r_{p, 2}-r_{p, 2}\right\|\right) & \ldots & \phi\left(\left\|r_{p, 2}-r_{p, n}\right\|\right) \\
\vdots & \vdots & \ddots & \vdots \\
\phi\left(\left\|r_{p, n}-r_{p, 1}\right\|\right) & \phi\left(\left\|r_{p, n}-r_{p, 2}\right\|\right) & \ldots & \phi\left(\left\|r_{p, n}-r_{p, n}\right\|\right)
\end{array}\right]_{n \times n} \quad \boldsymbol{P}_{p}=\left[\begin{array}{cccc}
1 & x_{p, 1} & y_{p, 1} & z_{p, 1} \\
1 & x_{p, 1} & y_{p, 1} & z_{p, 1} \\
\vdots & \vdots & \vdots \\
1 & x_{p, n} & y_{p, n} & z_{p, n}
\end{array}\right]_{n \times 4}
$$

The coefficients $\boldsymbol{a}$ and $\boldsymbol{\beta}$ can be solved based on Eq. (15). The displacements for the stiffener element nodes are then expressed as: 


$$
\begin{aligned}
\left\{\boldsymbol{u}_{s}\right\}=\left[\begin{array}{ll}
\boldsymbol{P}_{s} & \boldsymbol{\Phi}_{s p}
\end{array}\right]\left\{\begin{array}{l}
\boldsymbol{\beta} \\
\boldsymbol{a}
\end{array}\right\} & =\left[\begin{array}{ll}
\boldsymbol{P}_{s} & \boldsymbol{\Phi}_{s p}
\end{array}\right]\left[\begin{array}{cc}
\mathbf{0} & \boldsymbol{P}_{p}^{T} \\
\boldsymbol{P}_{p} & \boldsymbol{\Phi}_{p p}
\end{array}\right]^{-1}\left[\boldsymbol{T}_{p}\right]\left\{\boldsymbol{u}_{p}\right\} \\
& =\left[\boldsymbol{H}_{s p}\right]\left\{\boldsymbol{u}_{p}\right\}
\end{aligned}
$$

where

$$
\boldsymbol{\Phi}_{s p}=\left[\begin{array}{cccc}
\phi\left(\left\|r_{s, 1}-r_{p, 1}\right\|\right) & \phi\left(\left\|r_{s, 1}-r_{p, 2}\right\|\right) & \ldots & \phi\left(\left\|r_{s, 1}-r_{p, n}\right\|\right) \\
\phi\left(\left\|r_{s, 2}-r_{p, 1}\right\|\right) & \phi\left(\left\|r_{s, 2}-r_{p, 2}\right\|\right) & \ldots & \phi\left(\left\|r_{s, 2}-r_{p, n}\right\|\right) \\
\vdots & \vdots & \ddots & \vdots \\
\phi\left(\left\|r_{s, m}-r_{p, 1}\right\|\right) & \phi\left(\left\|r_{s, m}-r_{p, 2}\right\|\right) & \ldots & \phi\left(\left\|r_{s, m}-r_{p, n}\right\|\right)
\end{array}\right]_{m \times n} \quad \boldsymbol{P}_{s}=\left[\begin{array}{cccc}
1 & x_{s, 1} & y_{s, 1} & z_{s, 1} \\
1 & x_{s, 1} & y_{s, 1} & z_{s, 1} \\
\vdots & \vdots & \vdots \\
1 & x_{s, m} & y_{s, m} & z_{s, m}
\end{array}\right]_{m \times 4}
$$

$m$ is the number of stiffener element nodes and $\left[\boldsymbol{T}_{p}\right]$ in Eq. 16 is an intermediate matrix, whose expression is:

$$
\boldsymbol{T}_{p}=\left[\begin{array}{cccc}
0 & 0 & \cdots & 0 \\
0 & 0 & \cdots & 0 \\
0 & 0 & \cdots & 0 \\
0 & 0 & \cdots & 0 \\
1 & 0 & \cdots & 0 \\
0 & 1 & \cdots & 0 \\
\vdots & \vdots & \ddots & \vdots \\
0 & 0 & \cdots & 1
\end{array}\right]_{(n+4) \times n}
$$

Equation (16) shows the stiffener nodal displacement approximation in terms of the plate element nodal displacement using radial basis functions. In this study, each stiffener element node has five degrees of freedom, the expression for $N_{s p}$ as given in Eqs. (1c), 2c), (6) and (8) is: 


$$
\boldsymbol{N}_{s p}=\left[\begin{array}{ccccc}
\boldsymbol{H}_{s p} & 0 & 0 & 0 & 0 \\
0 & \boldsymbol{H}_{s p} & 0 & 0 & 0 \\
0 & 0 & \boldsymbol{H}_{s p} & 0 & 0 \\
0 & 0 & 0 & \boldsymbol{H}_{s p} & 0 \\
0 & 0 & 0 & 0 & \boldsymbol{H}_{s p}
\end{array}\right]
$$

where the expression for $\boldsymbol{H}_{s p}$ is given in Eq. 16.

\section{Verification Studies}

The model studied in a previous work is employed here for the approach verification [23]. A simply-supported square laminated panel with dimensions $0.30 \mathrm{~m} \times 0.30 \mathrm{~m}$ is considered. A symmetric composite laminated plate wherein each layer has tow-steered lamina with nonlinearly varying (NLV) fiber path orientations is considered. Four curvilinear stiffeners are generated by using the Hobby spline [30]. The geometric model of the stiffened plate is shown in Fig. 3a The stiffener width, $b_{s}$, equals the panel thickness, $t_{p}$. The material properties for both the laminated plate and the composite stiffeners are same and are given as: $E_{1}=181 \mathrm{GPa}, E_{2}=10.27 \mathrm{GPa}, G_{12}=7.17 \mathrm{GPa}, G_{13}=G_{23}=4 \mathrm{GPa}$, $v_{12}=0.28$ and density $\rho=1800 \mathrm{~kg} / \mathrm{m}^{3}$. The layer thickness is $1.272 \times 10^{-4} \mathrm{~m}$. The NLV fiber path orientation is defined in one of the quarters of the plate by using Lagrange Polynomials [31]. A total of 16-layer symmetric laminates with configuration of $\left[ \pm \Theta_{1}, \pm \Theta_{2}\right]_{2, s}$ are considered for the plate. The fiber path orientations in the $3 \times 3$ uniform reference points of the quarter plate, $\Theta_{1}$ and $\Theta_{2}$, are given in Appendix B.

A uniform end-shortening applied on the panel edges is considered: (a) $x=0, u=\Delta u_{0} / 2$ and (b) $x=a, u=-\Delta u_{0} / 2$ (see Fig. 3a where $a$ is the panel's length along the $x$-axis and $\Delta u_{0}=0.02 \mathrm{~mm}$. The other boundary conditions in pre-buckling analysis are: (a) $y=(0, b), v_{0}=0$ and (b) $w=0$ for four edges. Here, $b$ is the plate's width along the $y$-axis. For the buckling analysis, a simply-supported boundary condition is considered: $u=v=w=0$ for the four panel edges.

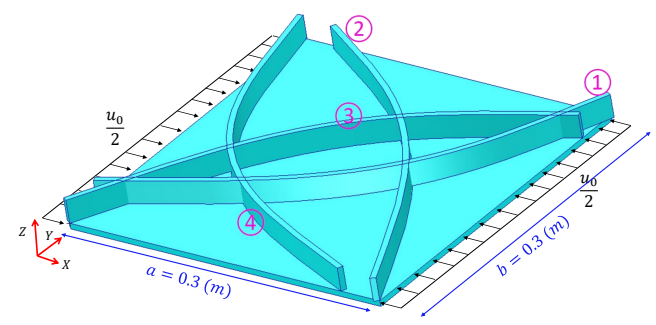

(a) CAD model

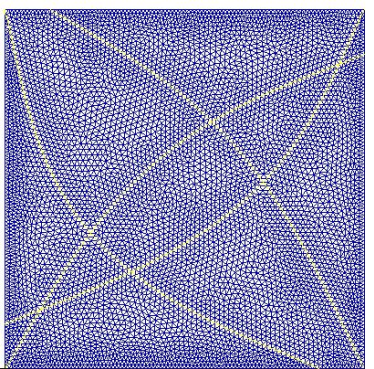

(b) NASTRAN mesh

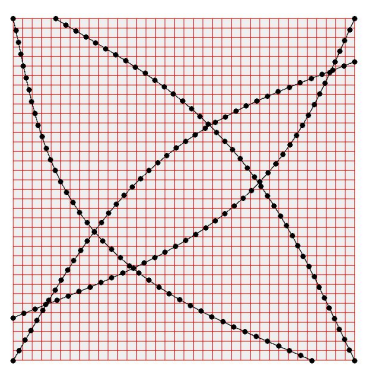

(c) Present mesh

Fig. 3 A square panel with NLV fiber path laminates and four arbitrarily shaped stiffeners 
The NASTRAN mesh and the present mesh are shown in Figs. 3b and 3c respectively. A fine mesh in NASTRAN is considered for obtaining converged results for the subsequent comparison. On the other hand, a fine mesh is normally used by EBF3PanelOpt [8] to avoid meshing failure. The composite plate is modeled by using the triangular elements (CTRIA3) in NASTRAN and the fiber path angle for each layer in the plate is evaluated at the center of each triangular element. The stiffeners are modeled as two-noded beam elements (CBEAM) in NASTRAN. The NASTRAN input files for the model are given in Appendix C. Both concentric and eccentric stiffeners are considered for these four curvilinear stiffeners. The eccentricity of the stiffener is defined by using the offset between the stiffener median plane and the plate median plane along the $z$-axis, $e$, as shown in Fig. 1 . In the present study, the eccentricity is $e=\frac{1}{2}\left(t_{p}+h_{s}\right)$ for eccentric stiffeners and it is zero for concentric stiffeners.

Although the first buckling mode is of interest to us, the first four buckling mode shapes are examined as shown in Figs. 6and 7] The present eigenvalues are compared against both the NASTRAN results and previous results [23]. The percentage differences between eigenvalues are computed and shown in a bracket below the present buckling mode shapes. The differences are compared against both NASTRAN results and previous results. Since MSC PATRAN only shows the magnitude of the eigenvectors computed from MSC NASTRAN, we exported NASTRAN results to MATLAB R2016b for plotting NASTRAN buckling mode shapes as seen in Figs. 6 and 7 The buckling mode shapes given by all the three approaches are in an excellent agreement to each other. The present buckling eigenvalues are within $1 \%$ and $3.5 \%$ of the previous results and the NASTRAN results, respectively. The first and second differences in the bracket in Figs. 6and 7 are compared to the NASTRAN result and the previous result, respectively. The slight differences between the present and the NASTRAN results can be attributed to the slightly different locations of the stiffeners in the two models. This is because NASTRAN has no Hobby spline for describing stiffener's shape and therefore a 10-noded B-spline in NASTRAN is used to approximate the stiffener's shape as close as possible to the one generated by the Hobby spline. Considering the $\mathrm{C}^{2}$ Wendland function is a positive definitive function, a unique interpolation is available. The buckling responses of the case where the linear terms are ignored in stiffener element node displacement in Eq. (11) are also studied, whose results are also shown in Figs. 6 and 7 There is no change in the buckling mode shapes but with slight differences in the buckling load factors comparing to the present results considering linear terms in Eq. (11). The buckling load factors are close to both previous and NASTRAN results.

The present approach of using radial basis function for computing the $N_{s p}$ matrix (see Eq. (8)) reduces the CPU time by around $20 \%$ as compared to the use of isoparametric shape functions, whereas the buckling mode shapes given by the two approaches are almost identical and the buckling eigenvalues are within $1 \%$. The slight difference between the buckling eigenvalues computed using the present and previous methods attribute to the different interpolations used in approximating the stiffener nodal displacement in terms of the plate element nodal displacements. When using the $C^{2}$ Wendland radial basis functions, the support radius (see Eq. (13) used in the present work is 2.2 times of element size. It means that each stiffener element stiffness is distributed to more plate elements than that only one plate element, 


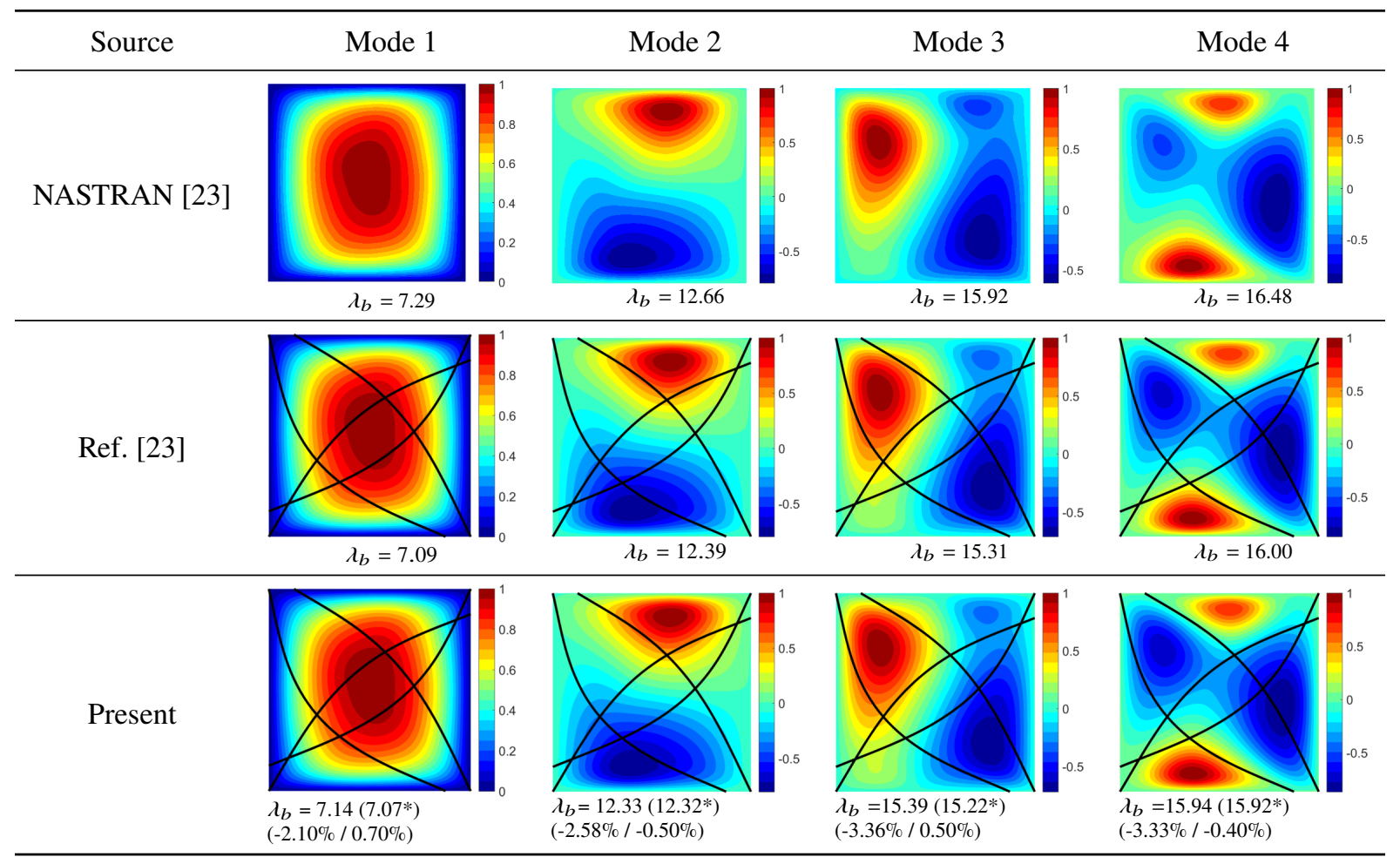

Fig. 4 Comparisons of buckling results for curvilinearly stiffened, VAT laminates with concentric stiffeners; * buckling results without considering linear terms, $p$, in Eq. (11)

Source Mode 1 Mode 2

Fig. 5 Comparisons of buckling results for curvilinearly stiffened, VAT laminates with eccentric stiffeners; * buckling results without considering linear terms, $p$, in Eq. 11] 
which is the case when using isoparametric shape function. It is these two fundamentally different approximations for the stiffener nodal displacements that cause the slight difference between the two buckling eigenvalues.

These results demonstrate that the present approach improves the computational efficiency of the buckling analysis of curvilinearly stiffened plates. Additionally, the present approach enables one to use a gradient based optimization for optimizing the stiffener's shape as the $C^{2}$ Wendland RBF has explicit expressions for its derivatives with respect to the stiffener's shape variables, such as the stiffener nodal coordinates. Additionally, the separate modeling allows one to use a fixed converged mesh plate, which enables optimizing lamination parameters for VAT laminates by using a gradient based optimization [32]. All in all, the present approach allows engineers to use gradient based optimization methods to optimize the stiffener's shape and the VAT laminates simultaneously for a further improvement in the structural performance.

\section{Conclusions}

This paper considers separate modelings for the plate and the individual stiffener for developing finite element models for curvilinearly stiffened plates. The stiffener displacements are approximated in terms of the plate nodal displacements by using $C^{2}$ continuous Wendland radial basis functions. This method avoids the need for determining the plate element IDs for each stiffener node a priori and avoids computing the natural coordinates of stiffener nodes from the high-order polynomials interpolations, used in the isoparametric shape functions, which was done in our previous work. Verification studies, on the buckling responses of curvilinearly stiffened variable-angle-tow laminates and subjected to in-plane shortening displacements, are conducted. It is shown that the present radial basis functions based approach of approximating the stiffener displacements for assembling curvilinear stiffeners stiffness matrices to those for the plate can accurately predict the buckling loads for the curvilinearly stiffened VAT laminates. The proposed approach is also found to require less CPU time in constructing the stiffener nodal displacements approximation matrix. The improved tool for structural analysis of curvilinearly stiffened plates by using the RBFs based stiffener displacement approximation approach can improve the optimization efficiency significantly compared to EBF3PanelOpt. Additionally, the global continuity of RBFs based stiffener nodal displacement approximation allows engineers to use gradient based optimization methods to optimize the stiffener's shape.

\section{Acknowledgement}

The authors thank anonymous reviewers for their insightful suggestions on improving the quality of this paper. 


\section{Appendix}

\section{A. Composite Beam Stiffness for Stiffeners}

$$
\boldsymbol{D}_{s}=\left[\begin{array}{ccccc}
\bar{C}_{11} A_{s} & 0 & 0 & \bar{C}_{11} A_{s} e_{s} & 0 \\
0 & \kappa \bar{C}_{66} A_{s} & 0 & 0 & \kappa \bar{C}_{66} A_{s} e_{s} \\
0 & 0 & \kappa \bar{C}_{55} A_{s} & 0 & 0 \\
\bar{C}_{11} A_{s} e_{s} & 0 & 0 & \bar{C}_{11} I_{n} & 0 \\
0 & \kappa \bar{C}_{66} A_{s} e_{s} & 0 & 0 & G J
\end{array}\right]
$$

where

$$
\begin{aligned}
& \bar{C}_{55}=C_{55}, \quad \bar{C}_{66}=C_{66} \\
& \bar{C}_{11}=\frac{C_{13}^{2} C_{22}-2 C_{12} C_{13} C_{23}+C_{11} C_{23}^{2}+C_{12}^{2} C_{33}-C_{11} C_{22} C_{33}}{C_{23}^{2}-C_{22} C_{33}}
\end{aligned}
$$

where $C_{i j}$ can be found in Ref. [33], $\kappa$ is the shear correction factor, 5/6. $A_{s}, e_{s}$ and $I_{n}$ are stiffener cross section area, stiffener eccentricity, $e_{s}=1 / 2\left(h_{s}+b_{s}\right)$ and the second area moment of inertia about $n$-axis (see Fig. 11), respectively. $G J$ is the equivalent torsional stiffness for the composite beam, which is computed based on the work by Nemeth [34].

\section{B. The fiber path angles, $T_{m n}$, at reference points for the plate}

The fiber path angles, $T_{m n}$, at $3 \times 3$ uniform reference points in the quarter plate studied in Section IV]are:

$$
\Theta_{1}: T_{m n}=\left[\begin{array}{ccc}
71 & 49.5 & 71.5 \\
67 & 50 & 51 \\
17 & 12 & 45
\end{array}\right] \text { degrees } \Theta_{2}: T_{m n}=\left[\begin{array}{ccc}
-72.5 & -59 & -59.5 \\
-65 & -54 & -50.5 \\
14 & 11.5 & 6
\end{array}\right] \text { degrees }
$$

The positive angle for the fiber ply orientation is obtained by rotating the global $x$-axis counterclockwise (see coordinate framework in Fig. 3a by a certain angle [23].

\section{NASTRAN input files}

NASTRAN input files for buckling analysis of stiffened, VAT laminated plates with nonlinear varying fiber paths under in-plane end-shortening for Section IV] are available in https://github.com/zhaowei0566/SPAD/tree/ master/AIAAJ_Paper_Data/prestressed_vibration_VAT_SP 


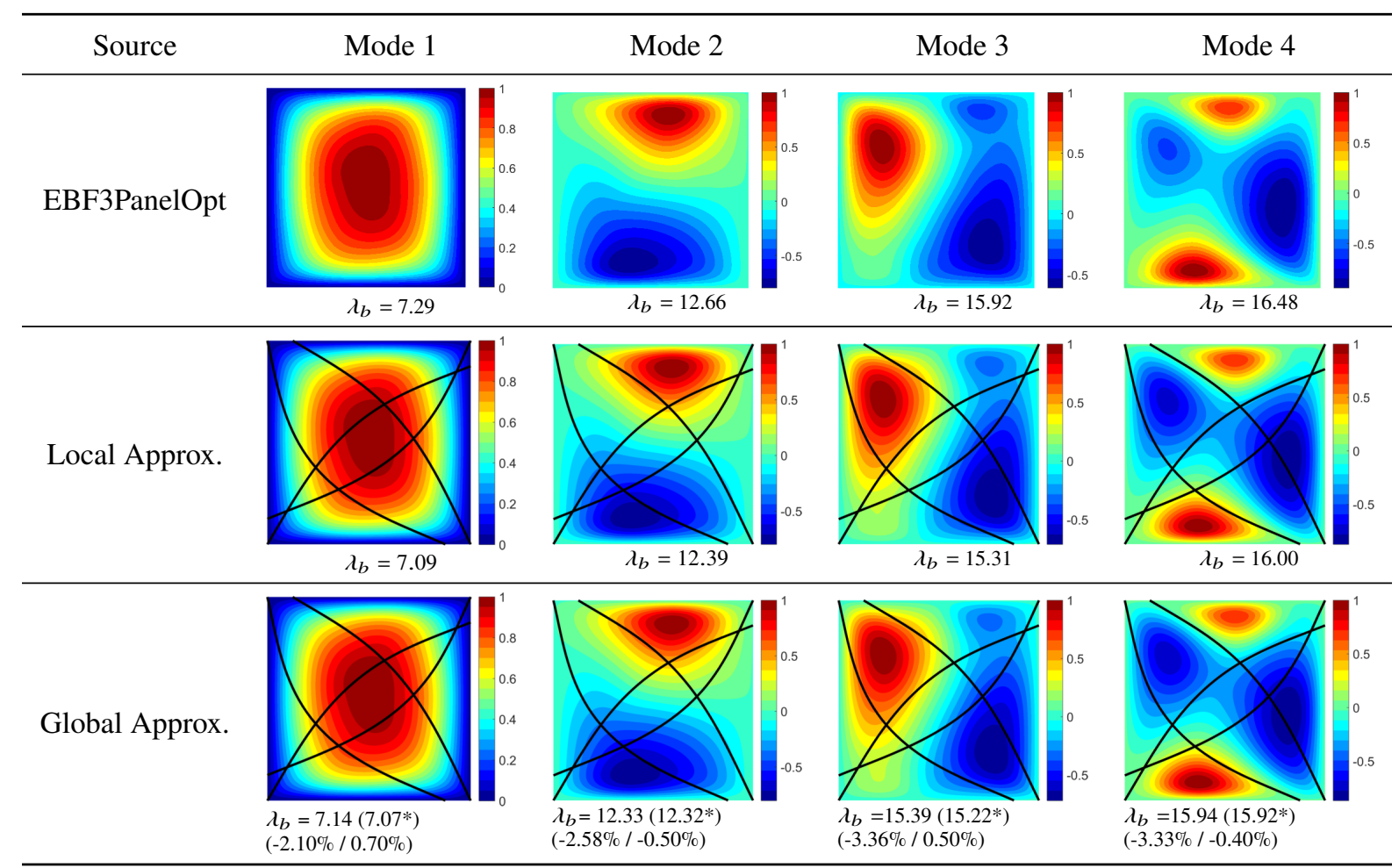

Fig. 6 Comparisons of buckling results for curvilinearly stiffened, VAT laminates with concentric stiffeners; * buckling results without considering linear terms, $p$, in Eq. 11]

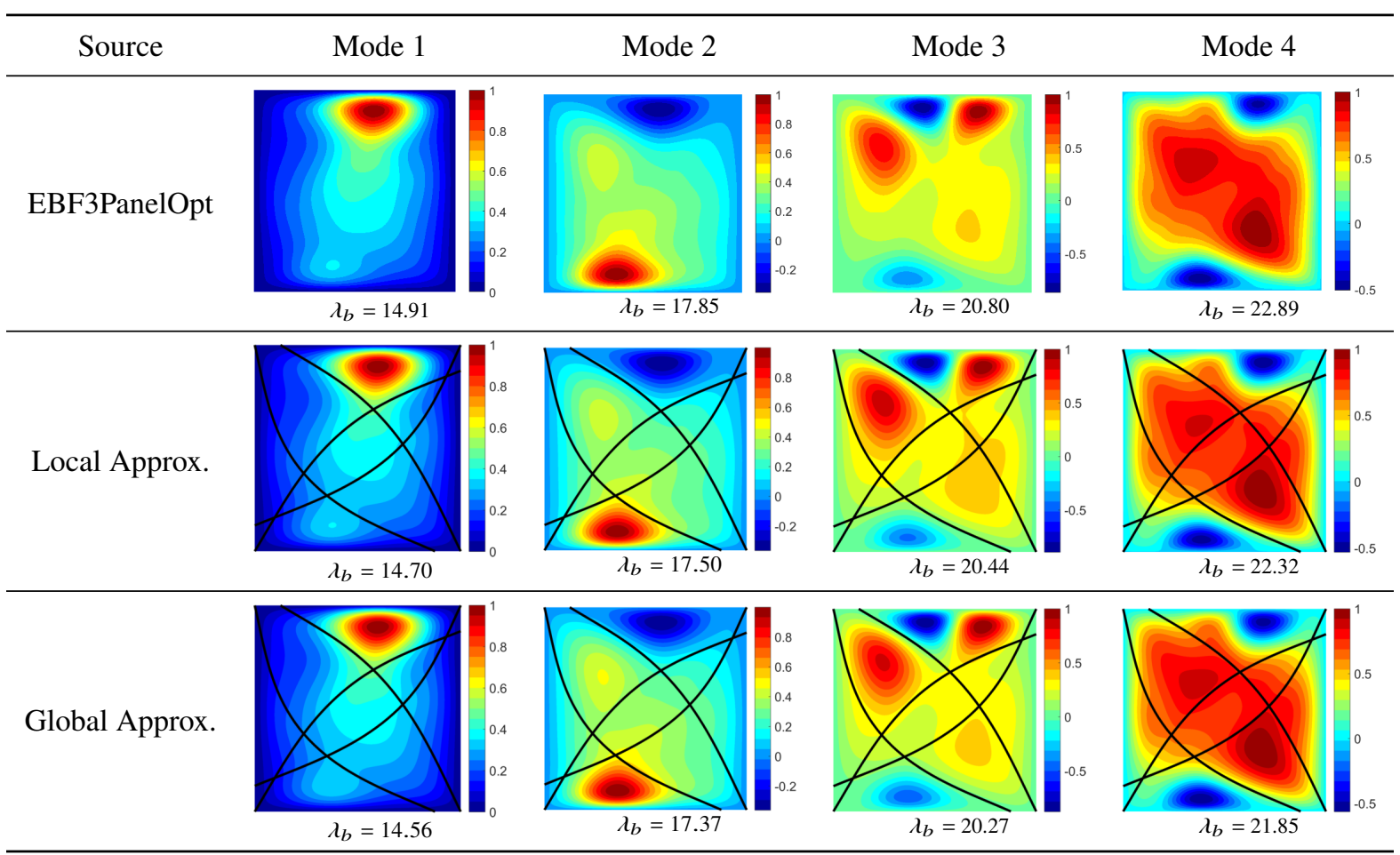

Fig. 7 Comparisons of buckling results for curvilinearly stiffened, VAT laminates with eccentric stiffeners; * buckling results without considering linear terms, $p$, in Eq. (11) 


\section{References}

[1] Zhao, W., and Kapania, R. K., "Buckling Analysis of Unitized Curvilinearly Stiffened Composite Panels," Composite Structures, Vol. 135, 2016, pp. 365-382. doi:10.1016/j.compstruct.2015.09.041.

[2] Zhao, W., and Kapania, R. K., "Vibration Analysis of Curvilinearly Stiffened Composite Panels Subjected to In-plane loads," AIAA Journal, Vol. 55, No. 3, 2017, pp. 981-997. doi:10.2514/1.J055047.

[3] Singh, K., Zhao, W., Jrad, M., and Kapania, R. K., "Hybrid Optimization of Curvilinearly Stiffened Shells Using Parallel Processing,” Journal of Aircraft, Vol. 56, No. 3, 2019, pp. 1068-1079. doi:10.2514/1.C035069.

[4] Stanford, B. K., and Jutte, C. V., "Comparison of Curvilinear Stiffeners and Tow Steered Composites for Aeroelastic Tailoring of Aircraft Wings," Computers and Structures, Vol. 183, 2017, pp. 48-60. doi:10.1016/j.compstruc.2017.01.010.

[5] Singh, K., and Kapania, R. K., "Buckling Load Maximization of Curvilinearly Stiffened Tow-Steered Laminates," Journal of Aircraft, Vol. 56, No. 6, 2019, pp. 2272-2284. doi:10.2514/1.C035358.

[6] Zhao, W., Singh, K., and Kapania, R. K., "Thermal Buckling Analysis and Optimization of Curvilinearly Stiffened Plates with Variable Angle Tow Laminates,” Journal of Spacecraft and Rockets, Vol. 54, No. 4, 2019, pp. 1-16. doi:10.2514/1.A34378.

[7] Kapania, R. K., Li, J., and Kapoor, H., “Optimal Design of Unitized Panels with Curvilinear Stiffeners," AIAA 5th ATIO and the AIAA 16th Lighter-than-Air Systems Technology Conference and Balloon Systems Conference, 2005, AIAA $2005-7482$. doi:10.2514/6.2005-7482.

[8] Mulani, S. B., Slemp, W. C., and Kapania, R. K., "EBF3PanelOpt: An Optimization Framework for Curvilinear Blade-stiffened Panels," Thin-Walled Structures, Vol. 63, 2013, pp. 13-26. doi:10.1016/j.tws.2012.09.008.

[9] Caffrey, J., and Lee, J. M., MSC/NASTRAN 2014 Linear Static Analysis: User's Guide, MacNeal-Schwendler Corp., Newport Beach, CA, 2014, pp. 640-658.

[10] Ahlbert, G., "Method Evaluation of Global-Local Finite Element Analysis,” Master's thesis, Linköping University, Department of Management and Engineering, Solid Mechanics, 2012. Linköping, Sweden.

[11] Tamijani, A. Y., and Kapania, R. K., "Vibration of Plate with Curvilinear Stiffeners using Mesh-free Method," AIAA Journal, Vol. 48, No. 8, 2010, pp. 1569-1581. doi:10.2514/1.43082.

[12] Tamijani, A. Y., and Kapania, R. K., "Buckling and Static Analysis of Curvilinearly Stiffened Plates using Mesh-free Method," AIAA Journal, Vol. 48, No. 12, 2010, pp. 2739-2751. doi:10.2514/1.43917.

[13] Tamijani, A. Y., and Kapania, R. K., "Chebyshev-Ritz Approach to Buckling and Vibration of Curvilinearly Stiffened Plate," AIAA Journal, Vol. 50, No. 5, 2012, pp. 1007-1018. doi:10.2514/1.J050042.

[14] Vescovini, R., Oliveri, V., Pizzi, D., Dozio, L., and Weaver, P., "Pre-buckling and Buckling Analysis of Variable-Stiffness, Curvilinearly Stiffened Panels,” Aerotecnica Missili \& Spazio, 2019, pp. 1-10. doi:10.1007/s42496-019-00031-4. 
[15] Vescovini, R., Oliveri, V., Pizzi, D., Dozio, L., and Weaver, P. M., “A Semi-analytical Approach for the Analysis of Variablestiffness Panels with Curvilinear Stiffeners," International Journal of Solids and Structures, Vol. 188, 2020, pp. 244-260. doi:10.1016/j.ijsolstr.2019.10.011.

[16] Alanbay, B., Singh, K., and Kapania, R. K., "Vibration of Curvilinearly Stiffened Plates Using Ritz Method With Orthogonal Jacobi Polynomials," ASME Journal of Vibration and Acoustics, Vol. 142, No. 1, 2020. doi:10.1115/1.4045098.

[17] Shi, P., Kapania, R. K., and Dong, C., "Vibration and Buckling Analysis of Curvilinearly Stiffened Plates Using Finite Element Method,” AIAA Journal, Vol. 53, No. 5, 2015, pp. 1319-1335. doi:10.2514/1.J053358.

[18] Shi, P., Kapania, R. K., and Dong, C., "Free Vibration of Curvilinearly Stiffened Shallow Shells," ASME Journal of Vibration and Acoustics, Vol. 137, No. 3, 2015, p. 031006. doi:10.1115/1.4029360.

[19] De Boer, A., Van der Schoot, M., and Bijl, H., "Mesh Deformation Based on Radial Basis Function Interpolation,” Computers and Structures, Vol. 85, No. 11-14, 2007, pp. 784-795. doi:10.1016/j.compstruc.2007.01.013.

[20] Zhao, W., Gupta, R., Kapania, R. K., and Schmidt, D. K., "Fuel Weight Minimization for Large N+3 Composite Transport with Multiple Control Surfaces," Application of MDO for Vehicle Design, AIAA SciTech Forum 2020, Orlando, FL, 2020. doi:10.2514/6.2020-0663, AIAA-2020-0663.

[21] Wei, P., Ma, H., and Wang, M. Y., "The Stiffness Spreading Method for Layout Optimization of Truss Structures," Structural and Multidisciplinary Optimization, Vol. 49, No. 4, 2014, pp. 667-682. doi:10.1007/s00158-013-1005-7.

[22] Byun, C., and Kapania, R. K., "Prediction of Interlaminar Stresses in Laminated Plates using Global Orthogonal Interpolation Polynomials," AIAA Journal, Vol. 30, No. 11, 1992, pp. 2740-2749. doi:10.2514/3.11293.

[23] Zhao, W., and Kapania, R. K., "Prestressed Vibration of Stiffened Variable-Angle Tow Laminated Plates," AIAA Journal, Vol. 57, No. 6, 2019, pp. 2575-2593. doi:10.2514/1.J057719.

[24] Beckert, A., and Wendland, H., "Multivariate Interpolation for Fluid-structure-interaction Problems using Radial Basis Functions," Aerospace Science and Technology, Vol. 5, No. 2, 2001, pp. 125-134. doi:10.1016/S1270-9638(00)01087-7.

[25] Buhmann, M. D., Radial Basis Functions: Theory and Implementations, Cambridge University Press, Cambridge, UK, 2003.

[26] Chen, C., Hon, Y., and Schaback, R., Scientific Computing with Radial Basis Functions, Department of Mathematics, University of Southern Mississippi, Hattiesburg, MS., 2007.

[27] Lonsain, J., “Aeroelastic Anaysis of a 3D Wing Structure with a Flexible Trailing Edge," Master's thesis, Delft University of Technology, 2017. Delft, The Netherland.

[28] Biancolini, M. E., Fast Radial Basis Functions for Engineering Applications, Springer, Switzerland, 2017, pp. 9-33.

[29] Wendland, H., "Piecewise Polynomial, Positive Definite and Compactly Supported Radial Functions of Minimal Degree," Advances in Computational Mathematics, Vol. 4, No. 1, 1995, pp. 389-396. doi:10.1007/BF02123482. 
[30] Hobby, J. D., “Smooth, Easy to Compute Interpolating Splines,” Discrete and Computational Geometry, Vol. 1, No. 2, 1986, pp. 123-140. doi:10.1007/BF02187690.

[31] Wu, Z., Weaver, P. M., Raju, G., and Kim, B. C., "Buckling Analysis And Optimisation Of Variable Angle Tow Composite Plates," Thin-walled Structures, Vol. 60, 2012, pp. 163-172. doi:10.1016/j.tws.2012.07.008.

[32] IJsselmuiden, S. T., Abdalla, M. M., and Gürdal, Z., "Optimization of Variable Stiffness Panels for Maximum Buckling Loads using Lamination Parameters," AIAA Journal, Vol. 48, No. 1, 2010, pp. 134-143.

[33] Reddy, J. N., "Mechanics of Laminated Composite Plates: Theory and Analysis, Second Edition,” CRC press, 2004 , pp. 132-142.

[34] Nemeth, M. P., "A Treatise on Equivalent-Plate Stiffnesses for Stiffened Laminated-Composite Plates and Plate-Like Lattices," NASA TP-2011-216882, 2011. 